\title{
Analysis of Non-polynomial Systems using the Sum of Squares Decomposition
}

\author{
Antonis Papachristodoulou ${ }^{1}$ and Stephen Prajna ${ }^{1}$ \\ Control and Dynamical Systems \\ California Institute of Technology \\ Pasadena, CA 91125, USA. \\ \{antonis,prajna\}@cds.caltech.edu
}

Summary. Recent advances in semidefinite programming along with use of the sum of squares decomposition to check nonnegativity have paved the way for efficient and algorithmic analysis of systems with polynomial vector fields. In this paper we present a systematic methodology for analyzing the more general class of non-polynomial vector fields, by recasting them into rational vector fields. The sum of squares decomposition techniques can then be applied in conjunction with an extension of the Lyapunov stability theorem to investigate the stability and other properties of the recasted systems, from which properties of the original, nonpolynomial systems can be inferred. This will be illustrated by some examples from the mechanical and chemical engineering domains.

\section{Introduction}

The analysis of nonlinear systems has always been a difficult task as the only direct, efficient methodology requires the construction of what is called a Lyapunov function. The difficulty lies not only in the 'manual' construction of Lyapunov functions but also in the complexity of testing the non-negativity of the two Lyapunov conditions. Indeed, even if someone was to propose a high order Lyapunov function, it might not at all be possible to verify the two conditions that it needs to satisfy: that it is positive definite in some region around the zero equilibrium and that its derivative along the system's trajectories is non-positive.

Recent advances in the areas of semidefinite programming and the use of the sum of squares decomposition to efficiently check nonnegativity have allowed an algorithmic procedure for systems analysis, something that was not possible before [10,9]. Despite these advances, this methodology is restricted to systems described by polynomial vector fields whereas physical systems, the functionality of which is in the focus of many research areas,

\footnotetext{
1 The authors contributed equally to this work.
} 
seldom have polynomial vector fields. For example, it is a common practice to use vector fields with non-rational powers to model enzymatic reactions in biological systems [7]. Also, the model of an aircraft in longitudinal flight contains trigonometric nonlinearities of the angle of attack and pitch angle, but in the same equations one usually captures the coefficients of lift and drag as polynomial descriptions of these variables. In this case the stability analysis of the closed loop system using the above methodology becomes difficult, as the same variable appears both in polynomial and non-polynomial terms. The same is true in the case of analysis of chemical processes, where the temperature appears in the energy equation both as a state and also exponentiated in Arrhenius law for the reaction rate.

It has been shown in [14] that any system with non-polynomial nonlinearities can be converted through a simple series of steps to a polynomial system with a larger state dimension, but with a series of equality constraints restricting the states to a manifold of the original state dimension. In some cases the recasting is 'exact', in the sense that the transformed system has a polynomial vector field with the same dimension as the original system consider for example the case

$$
\dot{x}(t)=c e^{-\alpha x(t)} .
$$

Setting $p(t)=c e^{-\alpha x(t)}$ we get immediately that

$$
\dot{p}(t)=-\alpha p^{2}(t) .
$$

In many cases, recasting increases the state dimension but equality constraints that arise from the recasting restrict the system to the original manifold. In particular, the constraints that arise can be either polynomial, or include nonpolynomial terms. Consider for example the case of a simple pendulum

$$
\frac{d}{d t}\left[\begin{array}{c}
\theta \\
\omega
\end{array}\right]=\left[\begin{array}{c}
\omega \\
-\frac{g}{l} \sin \theta
\end{array}\right]
$$

where $g$ is the gravitational constant, $l$ is the length of the pendulum, $\omega$ its angular velocity and $\theta$ the angular deviation of the bead from the vertical. Setting $x_{1}=\sin \theta$ and $x_{2}=\cos \theta$ one can easily rewrite the above system as

$$
\begin{aligned}
\frac{d}{d t}\left[\begin{array}{c}
x_{1} \\
\omega \\
x_{2}
\end{array}\right] & =\left[\begin{array}{c}
x_{2} \omega \\
-\frac{g}{l} x_{1} \\
-x_{1} \omega
\end{array}\right] \\
x_{1}^{2}+x_{2}^{2} & =1
\end{aligned}
$$

where the constraint $x_{1}^{2}+x_{2}^{2}=1$ is a polynomial equality in $\left(x_{1}, x_{2}\right)$ that restricts the 3 -D recasted system to the $2-\mathrm{D}$ evolution manifold.

However in some other cases, like the case of aircraft dynamics, the case of a reactor process, or the case of enzymatic reactions described by MichaelisMenten equations these equality constraints are not polynomial, e.g., one 
would have recastings of the form $x_{1}=\sin \alpha$, but then $x_{1}$ and $\alpha$ also appear in the equations. This is one particularly interesting case that we will explore.

The aim of this chapter is to address stability analysis of non-polynomial systems through a recasting as described above. We extend Lyapunov's stability theorem to handle recasted systems and then use the sum of squares decomposition to construct Lyapunov functions in the new coordinates. When mapped back to the original variables, these Lyapunov functions will contain the original non-polynomial terms.

In Section 2 we review briefly the Lyapunov stability theory for nonlinear systems and how the sum of squares decomposition can be used to construct Lyapunov functions. In Section 3 we present the recasting algorithm and extend the standard Lyapunov theorem to handle the recasted systems. In the examples section, Section 4, we present the analysis of four systems whose vector fields contain radical, trigonometric, irrational power, and exponential terms, which concludes the chapter.

\section{Background Material}

Systems analysis has been in the focus of research for many years. With the development of Lyapunov stability theory, the question of assessing stability of nonlinear systems through the properties of solutions to ordinary differential equations (ODEs) describing the systems was turned into a problem of the existence of what is now known as Lyapunov functions. This circumvented the problem of solving the ODE to prove stability. Nonetheless, no explicit algorithm was given on how these functions could be constructed.

The Lyapunov conditions require the construction of a positive definite function that is guaranteed to have non-positive derivative along the trajectories of the system. These conditions are inherently complex, as even testing non-negativity of a polynomial is NP-hard when the polynomial has degree 4 or higher [8]. A sufficient condition for checking non-negativity of a polynomial $p$ is to check whether it admits a sum of squares decomposition [10]; the latter is polynomial-time verifiable - it can be tested by solving a semidefinite programme (SDP).

In this section we give the background material on sum of squares and Lyapunov function theory that will be used in Section 3.

\subsection{The Sum of Squares Decomposition}

We will now give a brief introduction to sum of squares (SOS) polynomials and show how the existence of an SOS decomposition can be verified using semidefinite programming [16]. A more detailed description can be found in $[10,11]$ and the references therein. We also present briefly an extension of the S-procedure $[17,3]$ that is used in the main text. 
Definition 1. For $x \in \mathbb{R}^{n}$, a multivariate polynomial $p(x)$ is an SOS if there exist some polynomials $f_{i}(x), i=1 \ldots M$ such that $p(x)=\sum_{i=1}^{M} f_{i}^{2}(x)$.

An equivalent characterization of SOS polynomials is given in the following proposition.

Proposition 1. A polynomial $p(x)$ of degree $2 d$ is an SOS if and only if there exists a positive semidefinite matrix $Q$ and a vector of monomials $Z(x)$ containing all monomials in $x$ of degree $\leq d$ such that $p=Z(x)^{T} Q Z(x)$.

The proof of this proposition is based on the eigenvalue decomposition and can be found in [10]. In general, the monomials in $Z(x)$ are not algebraically independent. Expanding $Z(x)^{T} Q Z(x)$ and equating the coefficients of the resulting monomials to the ones in $p(x)$, we obtain a set of affine relations in the elements of $Q$. Since $p(x)$ being SOS is equivalent to $Q \geq 0$, the problem of finding a $Q$ which proves that $p(x)$ is an SOS can be cast as a semidefinite program (SDP). This was observed by Parrilo in [10].

Note that $p(x)$ being an SOS implies that $p(x) \geq 0$ for all $x \in \mathbb{R}^{n}$. However, the converse is not always true. Not all nonnegative polynomials can be written as SOS, apart from three special cases: (i) when $n=2$, (ii) when $\operatorname{deg}(p)=2$, and (iii) when $n=3$ and $\operatorname{deg}(p)=4$. See [13] for more details. Nevertheless, checking nonnegativity of $p(x)$ is an NP-hard problem when the degree of $p(x)$ is at least $4[8]$, whereas as argued in the previous paragraph, checking whether $p(x)$ can be written as an SOS is computationally tractable - it can be formulated as an SDP, which has worst-case polynomial time complexity. We will not entail in a discussion on how conservative the relaxation is, but there are several results suggesting that this is not too conservative $[13,11]$. Note that as the degree of $p(x)$ and/or its number of variables is increased, the computational complexity for testing whether $p(x)$ is an SOS increases. Nonetheless, the complexity overload is still a polynomial function of these parameters.

There is a close connection between sums of squares and robust control theory through Positivstellensatz, a central theorem in Real algebraic geometry [2]. This theorem allows us to formulate a hierarchy of polynomialtime computable stronger conditions [10] for the S-procedure type of analysis $[17,3]$. To see how we will be using this result say we want to use the S-procedure to check that the set:

$$
\left\{p(x) \geq 0 \text { when } p_{i}(x) \geq 0 \text { for } i=1, \ldots, n\right\}
$$

is non-empty. Instead of finding positive constant multipliers (the standard S-procedure), we search for $S O S$ multipliers $h_{i}(x)$ so that

$$
p(x)-\sum_{i} h_{i}(x) p_{i}(x) \text { is a SOS. }
$$

Since $h_{i}(x) \geq 0$ and condition (1) is satisfied, for any $x$ such that $p_{i}(x) \geq 0$ we automatically have $p(x) \geq 0$, so sufficiency follows. This condition is at least 
as powerful as the standard S-procedure, and many times it is strictly better; it is a special instance of positivstellensatz. By putting an upper bound on the degree of $h_{i}$ we can get a nested hierarchy of polynomial-time checkable conditions.

Besides this, what is more interesting is the case in which the monomials in the polynomial $p(x)$ have unknown coefficients, and we want to search for some values of those coefficients such that $p(x)$ is a sum of squares (and hence nonnegative). Since the unknown coefficients of $p(x)$ are related to the entries of $Q$ via affine constraints, it is evident that the search for the coefficients that make $p(x)$ an SOS can also be formulated as an SDP (these coefficients are themselves decision variables). This observation is crucial in the construction of Lyapunov functions and other S-procedure type multipliers.

Construction of an equivalent SDP for computing SOS decomposition as in Proposition 1 can be quite involved when the degree of the polynomials is high. For this reason, conversion of SOS conditions to the corresponding SDP has been automated in SOSTOOLS [12], a software package developed for this purpose. This software calls SeDuMi [15], an SDP solver to solve the resulting SDP, and converts the solutions back to the solutions of the original SOS programs. These software packages are used for solving all the examples in this chapter.

\subsection{Lyapunov Stability}

Here we concentrate on autonomous nonlinear systems of the form

$$
\dot{z}=f(z),
$$

where $z \in \mathbb{R}^{n}$ and for which we assume without loss of generality that $f(0)=$ 0 , i.e. the origin is an equilibrium of the system. One of the most important properties related to this equilibrium is its stability, and assessing whether stability of the equilibrium holds has been in the center of systems and control research for more than a century. It was not until just before the turn of the 19th century that A. M. Lyapunov formulated sufficient conditions for stability [18] that do not require knowledge of the solution, but are based on the construction of an 'energy-like' function, well known nowadays as a 'Lyapunov function'. Under some technical conditions, the existence of this function was later proved also necessary for asymptotic stability [5].

More precisely, the conditions are stated in the following theorem.

Theorem 1 (Lyapunov). For an open set $\mathcal{D} \subset \mathbb{R}^{n}$ with $0 \in \mathcal{D}$, suppose there exists a continuously differentiable function $V: \mathcal{D} \rightarrow \mathbb{R}$ such that

$$
\begin{aligned}
& V(0)=0, \\
& V(z)>0 \quad \forall z \in \mathcal{D} \backslash\{0\}, \\
& \frac{\partial V}{\partial z}(z) f(z) \leq 0 \quad \forall z \in \mathcal{D} .
\end{aligned}
$$


Then $z=0$ is a stable equilibrium of (2).

It is unfortunate that even with such a powerful theorem, the problem of proving stability of equilibria of nonlinear systems is still difficult; the reason is that there has been no coherent methodology for constructing the Lyapunov function $V(z)$.

In order to simplify the problem at hand, let us assume that $f(z)$ is a polynomial vector field, and that we will be searching for $V(z)$ that is also a polynomial in $z$. Then the two conditions in Theorem 1 become polynomial nonnegativity conditions. To circumvent the difficult task of testing them, we can restrict our attention to cases in which the two conditions admit SOS decompositions. This is the procedure that was originally pursued by Parrilo in his thesis [10]. For $\mathcal{D}=\mathbb{R}^{n}$, the conditions in Theorem 1 can then be formulated as SOS program stated in the following proposition, and a Lyapunov function that satisfies these conditions can be constructed using semidefinite programming.

Proposition 2. Suppose that for the system (2) there exists a polynomial function $V(z)$ such that

$$
\begin{gathered}
V(0)=0 \\
V(z)-\phi(z) \text { is } S O S, \\
-\frac{\partial V}{\partial z} f(z) \text { is SOS }
\end{gathered}
$$

where $\phi(z)>0$ for $z \neq 0$. Then the zero equilibrium of (2) is stable.

Proof. Condition (7) enforces $V(z)$ to be positive definite. Since condition (8) implies that $\dot{V}(z)$ is negative semidefinite, it follows that $V(z)$ is a Lyapunov function that proves stability of the origin.

In the above proposition, the function $\phi(z)$ is used to enforce positive definiteness of $V(z)$. If $V(z)$ is a polynomial of degree $2 d$, then $\phi(z)$ may be chosen as follows:

$$
\phi(z)=\sum_{i=1}^{n} \sum_{j=1}^{d} \epsilon_{i j} z_{i}^{2 j},
$$

where the $\epsilon$ 's satisfy

$$
\sum_{j=1}^{m} \epsilon_{i j}>\gamma \quad \forall i=1, \ldots, n,
$$

with $\gamma$ a positive number, and $\epsilon_{i j} \geq 0$ for all $i$ and $j$. In fact, this choice of $\phi(z)$ will force $V(z)$ to be radially unbounded, and hence the stability property holds globally if the conditions in Proposition 2 are met. 


\section{Recasting and Analysis of Recasted Systems}

\subsection{Recasting}

In this section we present an algorithm that can be used to convert a nonpolynomial system into a rational system. The algorithm is adapted from [14], and it is applicable to a very large class of non-polynomial systems, namely those whose vector field is composed of sums and products of elementary functions, or nested elementary functions of elementary functions. What are meant by elementary functions here are functions with explicit symbolic derivatives such as exponential $\left(e^{x}\right)$, logarithm $(\ln x)$, power $\left(x^{a}\right)$, trigonometric $(\sin x$, $\cos x$, etc.), and hyperbolic functions $(\sinh x, \cosh x$, etc.).

Suppose that the original system is given in the form

$$
\dot{z}_{i}=\sum_{j} \alpha_{j} \prod_{k} F_{i j k}(z),
$$

where $i=1, \ldots, n ; \alpha_{j}$ 's are real numbers; and $z=\left(z_{1}, \ldots, z_{n}\right)$. In the above equation, $F_{i j k}(z)$ are assumed to be elementary functions, or nested elementary functions of elementary functions. For the above system, the recasting algorithm is stated below.

\section{Algorithm 2 (adopted from [14], with some modifications)}

1. Let $x_{i}=z_{i}$, for $i=1, \ldots, n$.

2. For each $F_{i j k}(z)$ that is not of the form $F_{i j k}(z)=z_{\ell}^{a}$, where a is some integer and $1 \leq \ell \leq n$, introduce a new variable $x_{m}$. Define $x_{m}=F_{i j k}(z)$.

3. Compute the differential equation describing the time evolution of $x_{m}$ using the chain rule of differentiation.

4. Replace all appearances of such $F_{i j k}(z)$ in the system equations by $x_{m}$.

5. Repeat steps 2-4, until we obtain system equations with rational forms.

It is best to illustrate the application of the above algorithm by an example.

Example 1. Consider the differential equation

$$
\dot{z}=\sin (\exp (z)-1)+4 \ln \left(z^{2}+1\right),
$$

which we want to recast as a system with rational vector field. We start by defining $x_{1}=z, x_{2}=\sin (\exp (z)-1)$, and $x_{3}=\ln \left(z^{2}+1\right)$. By the chain rule of differentiation and replacing the appearances of $z, \sin (\exp (z)-1)$, and $\ln \left(z^{2}+1\right)$ in the resulting equations by $x_{1}, x_{2}$, and $x_{3}$, we obtain

$$
\begin{aligned}
\dot{x}_{1} & =x_{2}+4 x_{3}, \\
\dot{x}_{2} & =\cos (\exp (z)-1) \exp (z) \dot{z} \\
& =\cos \left(\exp \left(x_{1}\right)-1\right) \exp \left(x_{1}\right)\left(x_{2}+4 x_{3}\right), \\
\dot{x}_{3} & =\frac{2}{z^{2}+1} z \dot{z} \\
& =\frac{x_{1}\left(x_{2}+4 x_{3}\right)}{x_{1}^{2}+1} .
\end{aligned}
$$


Notice that the equations for $\dot{x}_{1}$ and $\dot{x}_{3}$ are in rational forms. However, the equation for $\dot{x}_{2}$ is not in a rational form and thus we continue by defining $x_{4}=\cos \left(\exp \left(x_{1}\right)-1\right)$ and $x_{5}=\exp \left(x_{1}\right)$. Using the chain rule of differentiation again, we obtain

$$
\begin{aligned}
\dot{x}_{2} & =x_{4} x_{5}\left(x_{2}+4 x_{3}\right), \\
\dot{x}_{4} & =-\sin \left(\exp \left(x_{1}\right)-1\right) \exp \left(x_{1}\right)\left(x_{2}+4 x_{3}\right) \\
& =-x_{2} x_{5}\left(x_{2}+4 x_{3}\right), \\
\dot{x}_{5} & =\exp \left(x_{1}\right)\left(x_{2}+4 x_{3}\right) \\
& =x_{5}\left(x_{2}+4 x_{3}\right) .
\end{aligned}
$$

At this point, we terminate the recasting process, since the differential equations describing the evolutions of $x_{1}, \ldots, x_{5}$ are already in rational forms.

More examples can be found in Section 4.

\subsection{Analysis}

The recasting process described in the previous subsection generally produces a recasted system whose dimension is higher than the dimension of the original system. To describe the original system faithfully, constraints of the form $x_{n+1}=F\left(x_{1}, \ldots, x_{n}\right)$ that are created when new variables are introduced (cf. Algorithm 2) should be taken into account. These constraints define an $n$-dimensional manifold on which the solutions to the original differential equations lie. In general such constraints cannot be converted into polynomial forms, even though sometimes there exist polynomial constraints that are induced by the recasting process. For example:

- Two variables introduced for trigonometric functions such as $x_{2}=\sin x_{1}$, $x_{3}=\cos x_{1}$ are constrained via $x_{2}^{2}+x_{3}^{2}=1$.

- Introducing a variable to replace a power function such as $x_{2}=\sqrt{x_{1}}$ induces the constraints $x_{2}^{2}-x_{1}=0, x_{2} \geq 0$.

- Introducing a variable to replace an exponential function such as $x_{2}=$ $\exp \left(x_{1}\right)$ induces the constraint $x_{2} \geq 0$.

We will shortly discuss how both types of constraints described above can be taken into account in the stability analysis using the sum of squares decomposition technique.

For our purpose, suppose that for a nonpolynomial system

$$
\dot{z}=f(z)
$$

which has an equilibrium at the origin, the recasted system obtained using the procedure of the previous subsection is written as

$$
\begin{aligned}
& \dot{\tilde{x}}_{1}=f_{1}\left(\tilde{x}_{1}, \tilde{x}_{2}\right), \\
& \dot{\tilde{x}}_{2}=f_{2}\left(\tilde{x}_{1}, \tilde{x}_{2}\right),
\end{aligned}
$$


where $\tilde{x}_{1}=\left(x_{1}, \ldots, x_{n}\right)=z$ are the state variables of the original system, $\tilde{x}_{2}=$ $\left(x_{n+1}, \ldots, x_{n+m}\right)$ are the new variables introduced in the recasting process, and $f_{1}\left(\tilde{x}_{1}, \tilde{x}_{2}\right), f_{2}\left(\tilde{x}_{1}, \tilde{x}_{2}\right)$ have rational forms.

We denote the constraints that arise directly from the recasting process by

$$
\tilde{x}_{2}=F\left(\tilde{x}_{1}\right),
$$

and those that arise indirectly by

$$
\begin{aligned}
& G_{1}\left(\tilde{x}_{1}, \tilde{x}_{2}\right)=0, \\
& G_{2}\left(\tilde{x}_{1}, \tilde{x}_{2}\right) \geq 0,
\end{aligned}
$$

where $F, G_{1}$, and $G_{2}$ are column vectors of functions with appropriate dimensions, and the equalities or inequalities hold entry-wise. The reader should keep in mind that constraints (13)-(14) are actually satisfied only when $\tilde{x}_{2}=F\left(\tilde{x}_{1}\right)$ are substituted to (13)-(14). Finally, denote the collective denominator of $f_{1}\left(\tilde{x}_{1}, \tilde{x}_{2}\right)$ and $f_{2}\left(\tilde{x}_{1}, \tilde{x}_{2}\right)$ by $g\left(\tilde{x}_{1}, \tilde{x}_{2}\right)$. That is, $g\left(\tilde{x}_{1}, \tilde{x}_{2}\right)$ should be a polynomial function such that $g\left(\tilde{x}_{1}, \tilde{x}_{2}\right) f_{1}\left(\tilde{x}_{1}, \tilde{x}_{2}\right)$ and $g\left(\tilde{x}_{1}, \tilde{x}_{2}\right) f_{2}\left(\tilde{x}_{1}, \tilde{x}_{2}\right)$ are polynomials. We also assume that $g\left(\tilde{x}_{1}, \tilde{x}_{2}\right)>0 \quad \forall\left(\tilde{x}_{1}, \tilde{x}_{2}\right) \in \mathcal{D}_{1} \times \mathcal{D}_{2}$, since otherwise the system is not well-posed.

Proving stability of the zero equilibrium of the original system (9) amounts to proving that all trajectories starting close enough to $z=0$ will remain close to this equilibrium point. This can be accomplished by finding a Lyapunov function $V(z)$ that satisfies the following conditions of Lyapunov's stability theorem, Theorem 1 . In terms of the new variables $\tilde{x}_{1}$ and $\tilde{x}_{2}$, sufficient conditions that guarantee the existence of a Lyapunov function for the original system are stated in the following proposition.

Proposition 3. Let $\mathcal{D}_{1} \subset \mathbb{R}^{n}$ and $\mathcal{D}_{2} \subset \mathbb{R}^{m}$ be open sets such that $0 \in \mathcal{D}_{1}$ and $F\left(\mathcal{D}_{1}\right) \subseteq \mathcal{D}_{2}$. Furthermore, define $\tilde{x}_{2,0}=F(0)$. If there exists a function $\tilde{V}: \mathcal{D}_{1} \times \mathcal{D}_{2} \rightarrow \mathbb{R}$ and column vectors of functions $\lambda_{1}\left(\tilde{x}_{1}, \tilde{x}_{2}\right), \lambda_{2}\left(\tilde{x}_{1}, \tilde{x}_{2}\right)$, $\sigma_{1}\left(\tilde{x}_{1}, \tilde{x}_{2}\right)$, and $\sigma_{2}\left(\tilde{x}_{1}, \tilde{x}_{2}\right)$ with appropriate dimensions such that

$$
\begin{aligned}
& \tilde{V}\left(0, \tilde{x}_{2,0}\right)=0 \\
& \tilde{V}\left(\tilde{x}_{1}, \tilde{x}_{2}\right)-\lambda_{1}^{T}\left(\tilde{x}_{1}, \tilde{x}_{2}\right) G_{1}\left(\tilde{x}_{1}, \tilde{x}_{2}\right)-\sigma_{1}^{T}\left(\tilde{x}_{1}, \tilde{x}_{2}\right) G_{2}\left(\tilde{x}_{1}, \tilde{x}_{2}\right) \ldots \\
& \geq \phi\left(\tilde{x}_{1}, \tilde{x}_{2}\right) \quad \forall\left(\tilde{x}_{1}, \tilde{x}_{2}\right) \in \mathcal{D}_{1} \times \mathcal{D}_{2} \\
&-g\left(\tilde{x}_{1}, \tilde{x}_{2}\right)\left(\frac{\partial \tilde{V}}{\partial \tilde{x}_{1}}\left(\tilde{x}_{1}, \tilde{x}_{2}\right) f_{1}\left(\tilde{x}_{1}, \tilde{x}_{2}\right)+\frac{\partial \tilde{V}}{\partial \tilde{x}_{2}}\left(\tilde{x}_{1}, \tilde{x}_{2}\right) f_{2}\left(\tilde{x}_{1}, \tilde{x}_{2}\right)\right) \ldots \\
&-\lambda_{2}^{T}\left(\tilde{x}_{1}, \tilde{x}_{2}\right) G_{1}\left(\tilde{x}_{1}, \tilde{x}_{2}\right)-\sigma_{2}^{T}\left(\tilde{x}_{1}, \tilde{x}_{2}\right) G_{2}\left(\tilde{x}_{1}, \tilde{x}_{2}\right) \ldots \\
& \geq 0 \quad \forall\left(\tilde{x}_{1}, \tilde{x}_{2}\right) \in \mathcal{D}_{1} \times \mathcal{D}_{2} \\
& \sigma_{1}\left(\tilde{x}_{1}, \tilde{x}_{2}\right) \geq 0 \quad \forall\left(\tilde{x}_{1}, \tilde{x}_{2}\right) \in \mathbb{R}^{n+m} \\
& \sigma_{2}\left(\tilde{x}_{1}, \tilde{x}_{2}\right) \geq 0 \quad \forall\left(\tilde{x}_{1}, \tilde{x}_{2}\right) \in \mathbb{R}^{n+m}
\end{aligned}
$$


for some scalar function $\phi\left(\tilde{x}_{1}, \tilde{x}_{2}\right)$ with $\phi\left(\tilde{x}_{1}, F\left(\tilde{x}_{1}\right)\right)>0 \quad \forall \tilde{x}_{1} \in \mathcal{D}_{1} \backslash\{0\}$, then $z=0$ is a stable equilibrium of (9).

Proof. Define $V(z)=\tilde{V}(z, F(z))$. From (15) it is straightforward to verify that (3) is satisfied by $V(z)$. Now, (13)-(14), (16), and (18) imply that

$$
\begin{aligned}
V\left(\tilde{x}_{1}, \tilde{x}_{2}\right) & \geq \phi\left(\tilde{x}_{1}, \tilde{x}_{2}\right)+\lambda_{1}^{T}\left(\tilde{x}_{1}, \tilde{x}_{2}\right) G_{1}\left(\tilde{x}_{1}, \tilde{x}_{2}\right)+\sigma_{1}^{T}\left(\tilde{x}_{1}, \tilde{x}_{2}\right) G_{2}\left(\tilde{x}_{1}, \tilde{x}_{2}\right) \\
& \geq \phi\left(\tilde{x}_{1}, \tilde{x}_{2}\right) \quad \forall\left(\tilde{x}_{1}, \tilde{x}_{2}\right) \in \mathcal{D}_{1} \times \mathcal{D}_{2} .
\end{aligned}
$$

Since $\phi(z, F(z))>0 \quad \forall z \in \mathcal{D}_{1} \backslash\{0\}$ and $F\left(\mathcal{D}_{1}\right) \subseteq \mathcal{D}_{2}$, it follows that $V(z)>$ $0 \forall z \in \mathcal{D}_{1} \backslash\{0\}$, hence (4) is satisfied.

Finally, by the chain rule of differentiation we have

$$
\frac{\partial V}{\partial z}(z) f(z)=\frac{\partial \tilde{V}}{\partial \tilde{x}_{1}}(z, F(z)) f_{1}(z, F(z))+\frac{\partial \tilde{V}}{\partial \tilde{x}_{2}}(z, F(z)) f_{2}(z, F(z)),
$$

and using the same argument as above in conjunction with (13)-(14), (17), (19), and the fact that $g\left(\tilde{x}_{1}, \tilde{x}_{2}\right)>0$, we see that the condition (5) is also satisfied.

Since the conditions (3)-(5) are fulfilled by $V(z)$, we conclude that $V(z)$ is a Lyapunov function for (9) and therefore $z=0$ is a stable equilibrium of the system.

The above non-negativity conditions can be relaxed to appropriate sum of squares conditions so that they can be algorithmically verified using semidefinite programming, as discussed in Section 2. This will also lead the way to an algorithmic construction of the Lyapunov function $V$. Here we assume that $\mathcal{D}_{1} \times \mathcal{D}_{2}$ is a semialgebraic set described by the following inequalities:

$$
\mathcal{D}_{1} \times \mathcal{D}_{2}=\left\{\left(\tilde{x}_{1}, \tilde{x}_{2}\right) \in \mathbb{R}^{n} \times \mathbb{R}^{m}: G_{\mathcal{D}}\left(\tilde{x}_{1}, \tilde{x}_{2}\right) \geq 0\right\},
$$

where $G_{\mathcal{D}}\left(\tilde{x}_{1}, \tilde{x}_{2}\right)$ is a column vector of polynomials and the inequality is satisfied entry-wise. With all this notation, the sum of squares conditions can be stated as follows.

Proposition 4. Let the system (10)-(11) and the functions $F\left(\tilde{x}_{2}\right), G_{1}\left(\tilde{x}_{1}, \tilde{x}_{2}\right)$, $G_{2}\left(\tilde{x}_{1}, \tilde{x}_{2}\right), G_{\mathcal{D}}\left(\tilde{x}_{1}, \tilde{x}_{2}\right)$, and $g\left(\tilde{x}_{1}, \tilde{x}_{2}\right)$ be given. Define $\tilde{x}_{2,0}=F(0)$. If there exists a polynomial function $\tilde{V}\left(\tilde{x}_{1}, \tilde{x}_{2}\right)$, column vectors of polynomial functions $\lambda_{1}\left(\tilde{x}_{1}, \tilde{x}_{2}\right), \lambda_{2}\left(\tilde{x}_{1}, \tilde{x}_{2}\right)$, and column vectors of sum of squares polynomials $\sigma_{1}\left(\tilde{x}_{1}, \tilde{x}_{2}\right), \sigma_{2}\left(\tilde{x}_{1}, \tilde{x}_{2}\right), \sigma_{3}\left(\tilde{x}_{1}, \tilde{x}_{2}\right), \sigma_{4}\left(\tilde{x}_{1}, \tilde{x}_{2}\right)$ with appropriate dimensions such that 


$$
\begin{aligned}
\tilde{V}\left(0, \tilde{x}_{2,0}\right) & =0 \\
\tilde{V}\left(\tilde{x}_{1}, \tilde{x}_{2}\right) & -\lambda_{1}^{T}\left(\tilde{x}_{1}, \tilde{x}_{2}\right) G_{1}\left(\tilde{x}_{1}, \tilde{x}_{2}\right)-\sigma_{1}^{T}\left(\tilde{x}_{1}, \tilde{x}_{2}\right) G_{2}\left(\tilde{x}_{1}, \tilde{x}_{2}\right) \ldots \\
& -\sigma_{3}^{T}\left(\tilde{x}_{1}, \tilde{x}_{2}\right) G_{\mathcal{D}}\left(\tilde{x}_{1}, \tilde{x}_{2}\right)-\phi\left(\tilde{x}_{1}, \tilde{x}_{2}\right) \text { is a sum of squares, } \\
-g\left(\tilde{x}_{1}, \tilde{x}_{2}\right) & \left(\frac{\partial \tilde{V}}{\partial \tilde{x}_{1}}\left(\tilde{x}_{1}, \tilde{x}_{2}\right) f_{1}\left(\tilde{x}_{1}, \tilde{x}_{2}\right)+\frac{\partial \tilde{V}}{\partial \tilde{x}_{2}}\left(\tilde{x}_{1}, \tilde{x}_{2}\right) f_{2}\left(\tilde{x}_{1}, \tilde{x}_{2}\right)\right) \ldots \\
& -\lambda_{2}^{T}\left(\tilde{x}_{1}, \tilde{x}_{2}\right) G_{1}\left(\tilde{x}_{1}, \tilde{x}_{2}\right)-\sigma_{2}^{T}\left(\tilde{x}_{1}, \tilde{x}_{2}\right) G_{2}\left(\tilde{x}_{1}, \tilde{x}_{2}\right) \ldots \\
& -\sigma_{4}^{T}\left(\tilde{x}_{1}, \tilde{x}_{2}\right) G_{\mathcal{D}}\left(\tilde{x}_{1}, \tilde{x}_{2}\right) \text { is a sum of squares }
\end{aligned}
$$

for some scalar polynomial function $\phi\left(\tilde{x}_{1}, \tilde{x}_{2}\right)$ with $\phi\left(\tilde{x}_{1}, F\left(\tilde{x}_{1}\right)\right)>0 \quad \forall \tilde{x}_{1} \in$ $\mathcal{D}_{1} \backslash\{0\}$, then $z=0$ is a stable equilibrium of $(9)$.

Proof. We will show that the above conditions imply the conditions in Proposition 3. Since $\sigma_{1}\left(\tilde{x}_{1}, \tilde{x}_{2}\right), \sigma_{2}\left(\tilde{x}_{1}, \tilde{x}_{2}\right)$ are sums of squares, (18)-(19) automati-

cally hold. It remains to show that (21)-(22) imply (16)-(17). The condition that $(21)$ is a sum of squares implies that

$$
\begin{aligned}
\tilde{V}\left(\tilde{x}_{1}, \tilde{x}_{2}\right) & -\lambda_{1}^{T}\left(\tilde{x}_{1}, \tilde{x}_{2}\right) G_{1}\left(\tilde{x}_{1}, \tilde{x}_{2}\right)-\sigma_{1}^{T}\left(\tilde{x}_{1}, \tilde{x}_{2}\right) G_{2}\left(\tilde{x}_{1}, \tilde{x}_{2}\right) \\
& \geq \sigma_{3}^{T}\left(\tilde{x}_{1}, \tilde{x}_{2}\right) G_{\mathcal{D}}\left(\tilde{x}_{1}, \tilde{x}_{2}\right)+\phi\left(\tilde{x}_{1}, \tilde{x}_{2}\right)
\end{aligned}
$$

Now, for $\left(\tilde{x}_{1}, \tilde{x}_{2}\right) \in \mathcal{D}_{1} \times \mathcal{D}_{2}$, we have $\sigma_{3}\left(\tilde{x}_{1}, \tilde{x}_{2}\right) G_{\mathcal{D}}\left(\tilde{x}_{1}, \tilde{x}_{2}\right) \geq 0$ and therefore it follows that for any such $\left(\tilde{x}_{1}, \tilde{x}_{2}\right)$,

$$
\tilde{V}\left(\tilde{x}_{1}, \tilde{x}_{2}\right)-\lambda_{1}^{T}\left(\tilde{x}_{1}, \tilde{x}_{2}\right) G_{1}\left(\tilde{x}_{1}, \tilde{x}_{2}\right)-\sigma_{1}^{T}\left(\tilde{x}_{1}, \tilde{x}_{2}\right) G_{2}\left(\tilde{x}_{1}, \tilde{x}_{2}\right) \geq \phi\left(\tilde{x}_{1}, \tilde{x}_{2}\right),
$$

which is (16). Using the same argument as above, it is straightforward to show that (17) is also fulfilled.

\section{Examples}

Here we present four examples. In the first example the vector field of the system includes a radical term. Such terms appear frequently when considering systems with saturation nonlinearities. The second example is a mechanical system, whose description contains trigonometric terms that appear when considering the system in cylindric coordinates. The third example shows how one can analyze non-polynomial vector fields with irrational powers, which for example appear in models of biological systems. In the last example we analyze a system that appears frequently in chemical engineering, that of a diabatic Continuous Stirred Tank Reactor (CSTR) with a single first-order exothermic irreversible reaction $A \rightarrow B$.

\subsection{Example 4.1: System with Saturation Nonlinearity}

For a general system, finding a global Lyapunov function is difficult, as one of polynomial form in the variables considered might not exist. However, if 
a term is expected to appear in a Lyapunov function, the search can be directed to include that term in the Lyapunov function expression sought, by changing variables and recasting the system in an equivalent one in terms of that variable, making use of inequality and equality constraints.

Consider, for example, the system

$$
\begin{aligned}
& \dot{x}_{1}=x_{2}, \\
& \dot{x}_{2}=-\varphi\left(x_{1}+x_{2}\right),
\end{aligned}
$$

where the function $\varphi$ is a saturation function of the following form:

$$
\varphi(\sigma)=\frac{\sigma}{\sqrt{1+\sigma^{2}}}
$$

This function has only one equilibrium point, namely the origin. Notice that for the above memoryless nonlinearity,

$$
\Phi(\sigma)=\int_{0}^{\sigma} \varphi(\tau) d \tau=\sqrt{1+\sigma^{2}}-1
$$

is a positive definite function. In fact, we expect terms of the above form to appear in the Lyapunov function.

First rewrite the above system in a polynomial form, as it has nonpolynomial terms. For this purpose, introduce the following auxiliary variables:

$$
\begin{aligned}
& u_{1}=\sqrt{1+\left(x_{1}+x_{2}\right)^{2}}, \\
& u_{2}=1 / u_{1}, \\
& u_{3}=\sqrt{1+x_{1}^{2}}, \\
& u_{4}=1 / u_{3} .
\end{aligned}
$$

Then the equations of motion for the above system become

$$
\begin{aligned}
& \dot{x}_{1}=x_{2}, \\
& \dot{x}_{2}=-\left(x_{1}+x_{2}\right) u_{2}, \\
& \dot{u}_{1}=\left(x_{1}+x_{2}\right)\left(x_{2}-x_{1} u_{2}-x_{2} u_{2}\right) u_{2}, \\
& \dot{u}_{2}=-\left(x_{1}+x_{2}\right)\left(x_{2}-x_{1} u_{2}-x_{2} u_{2}\right) u_{2}^{3}, \\
& \dot{u}_{3}=x_{1} x_{2} u_{4}, \\
& \dot{u}_{4}=-x_{1} x_{2} u_{4}^{3} .
\end{aligned}
$$

In addition, we have a number of equality and inequality constraints

$$
\begin{aligned}
u_{1}^{2} & =1+\left(x_{1}+x_{2}\right)^{2}, \\
u_{1} u_{2} & =1, \\
u_{3}^{2} & =1+x_{1}^{2}, \\
u_{3} u_{4} & =1, \\
u_{i} & \geq 0, \quad \text { for } i=1,2,3,4 .
\end{aligned}
$$


Now the system is in the form (10)-(11) with $\tilde{x}_{1}=\left(x_{1}, x_{2}\right)$ and $\tilde{x}_{2}=$ $\left(u_{1}, u_{2}, u_{3}, u_{4}\right)$. The above constraints correspond to Equation (12). The new representation allows us to use SOS decomposition to compute a Lyapunov function for this problem, using Proposition 4. Terms that are nonpolynomial in $x_{1}$ and $x_{2}$, such as $u_{3}$, can be included in the search, and indeed they should be, as they have the same form as the positive definite function (25). Thus, for example, we may search for a Lyapunov function of the following form:

$$
V=a_{1}+a_{2} u_{3}+a_{3} x_{1}^{2}+a_{4} x_{1} x_{2}+a_{5} x_{2}^{2},
$$

where the $a_{i}$ 's are the unknowns, with $a_{1}+a_{2}=0$, so that $V$ is equal to zero at $\left(x_{1}, x_{2}\right)=(0,0)$. To guarantee positive definiteness, we require $V$ to satisfy

$$
\left(V-\epsilon_{1}\left(u_{3}-1\right)-\epsilon_{2} x_{1}^{2}-\epsilon_{3} x_{2}^{2}\right) \text { is a sum of squares, }
$$

with $\epsilon_{1}, \epsilon_{2}, \epsilon_{3}$ being non-negative decision variables that satisfy, for example,

$$
\begin{aligned}
\epsilon_{1}+\epsilon_{2} & \geq 0.1 \\
\epsilon_{3} & \geq 0.1
\end{aligned}
$$

Using this method, a Lyapunov function has been constructed for the system:

$$
\begin{aligned}
V & =-3.9364+3.9364 u_{3}+0.0063889 x_{1}^{2}+0.010088 x_{1} x_{2}+2.0256 x_{2}^{2} \\
& =-3.9364+3.9364 \sqrt{1+x_{1}^{2}}+0.0063889 x_{1}^{2}+0.010088 x_{1} x_{2}+2.0256 x_{2}^{2} .
\end{aligned}
$$

The level curves of this Lyapunov function are shown in Figure 1.

\subsection{Example 4.2: Whirling Pendulum}

Consider the whirling pendulum [4] shown in Figure 2. It is a pendulum of length $l_{p}$ whose suspension end is attached to a rigid arm of length $l_{a}$, with a mass $m_{b}$ attached to its free end. The arm rotates with angular velocity $\dot{\theta}_{a}$. The pendulum can oscillate with angular velocity $\dot{\theta}_{p}$ in a plane normal to the arm, making an angle $\theta_{p}$ with the vertical in the instantaneous plane of motion. We will ignore frictional effects and assume that all links are slender so that their moment of inertia can be neglected.

Using $x_{1}=\theta_{p}$ and $x_{2}=\dot{\theta}_{p}$ as state variables, we obtain the following state equations for the system:

$$
\begin{aligned}
& \dot{x}_{1}=x_{2}, \\
& \dot{x}_{2}=\dot{\theta}_{a}^{2} \sin x_{1} \cos x_{1}-\frac{g}{l_{p}} \sin x_{1} .
\end{aligned}
$$

The number and stability properties of equilibria in this system depend on the value of $\dot{\theta}_{a}$. When the condition 


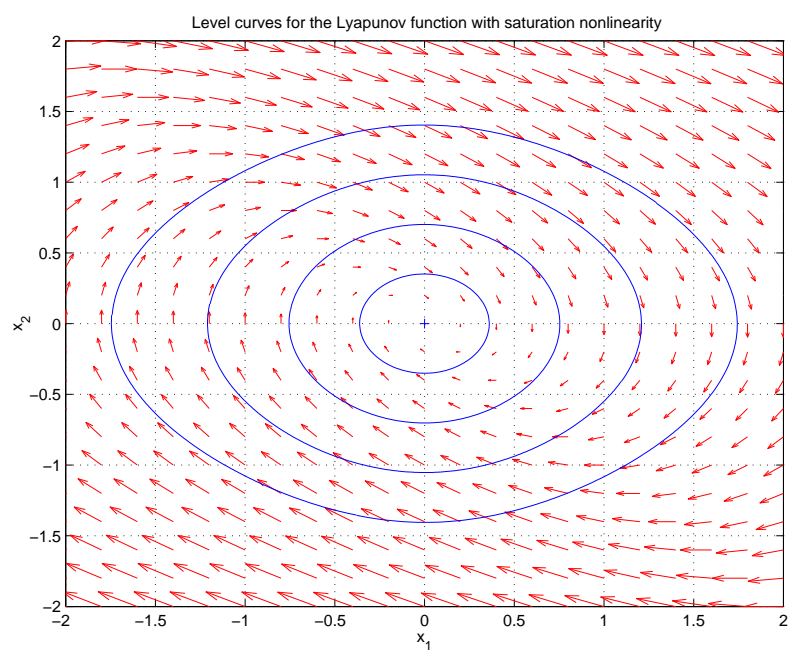

Fig. 1. Global Lyapunov function for the system with saturation nonlinearity. Arrows show vector field, solid lines show level curves of the Lyapunov function.

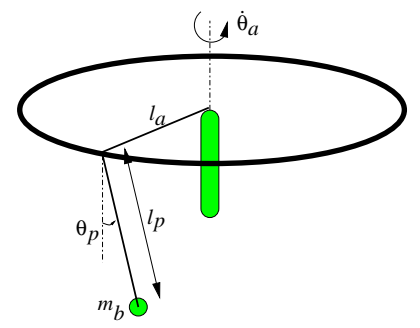

Fig. 2. The whirling pendulum

$$
\dot{\theta}_{a}^{2}<g / l_{p}
$$

is satisfied, the only equilibria in the system are $\left(x_{1}, x_{2}\right)$ satisfying $\sin x_{1}=0$, $x_{2}=0$. One equilibrium corresponds to $x_{1}=0$, i.e., the pendulum is hanging vertically downward (stable), and the other equilibrium corresponds to $x_{1}=\pi$, i.e., the vertically upward position (unstable). As $\dot{\theta}_{a}^{2}$ is increased beyond $g / l_{p}$, a supercritical pitchfork bifurcation of equilibria occurs [6]. The $\left(x_{1}, x_{2}\right)=(0,0)$ equilibrium becomes unstable, and two other equilibria appear. These equilibria correspond to $\cos x_{1}=\frac{g}{l_{p} \dot{\theta}_{a}^{2}}, x_{2}=0$.

We will now prove the stability of the equilibrium point at the origin for $\dot{\theta}_{a}$ satisfying (28), by constructing a Lyapunov function. Obviously the energy of this mechanical system can be used as a Lyapunov function, but since our purpose is to show that a Lyapunov function can be found using the SOS decomposition, we will assume that our knowledge is limited to the 
state equations describing the system and that we know nothing about the underlying energy.

Since the vector field (26)-(27) is not polynomial, a transformation to a polynomial vector field must be performed before we are able to construct a Lyapunov function using the SOS decomposition. For this purpose, introduce $u_{1}=\sin x_{1}$ and $u_{2}=\cos x_{1}$ to get:

$$
\begin{aligned}
& \dot{x}_{1}=x_{2}, \\
& \dot{x}_{2}=\dot{\theta}_{a}^{2} u_{1} u_{2}-\frac{g}{l_{p}} u_{1}, \\
& \dot{u}_{1}=x_{2} u_{2}, \\
& \dot{u}_{2}=-x_{2} u_{1} .
\end{aligned}
$$

In addition, we have the algebraic constraint

$$
u_{1}^{2}+u_{2}^{2}-1=0 .
$$

The whirling pendulum system will now be described by Equations (29)-(33). Notice that all the functions here are polynomial, so that Proposition 4 can be used to prove stability.

We will perform the analysis with the parameters of the system set at some fixed values. Assume that all the parameters except $g$ are equal to 1 , and $g$ itself is equal to 10 , for which condition (28) is satisfied. For a mechanical system like this, we expect that some trigonometric terms will be needed in the Lyapunov function. Thus we will try to find a Lyapunov function of the following form:

$$
\begin{aligned}
V & =a_{1} x_{2}^{2}+a_{2} u_{1}^{2}+a_{3} u_{2}^{2}+a_{4} u_{2}+a_{5}, \\
& =a_{1} x_{2}^{2}+a_{2} \sin ^{2} x_{1}+a_{3} \cos ^{2} x_{1}+a_{4} \cos x_{1}+a_{5}
\end{aligned}
$$

where the $a_{i}$ 's are the unknown coefficients. These coefficients must satisfy

$$
a_{3}+a_{4}+a_{5}=0,
$$

for $V$ to be equal to zero at $\left(x_{1}, x_{2}\right)=(0,0)$. To guarantee that $V$ is positive definite, we search for $V$ s that satisfy

$$
V-\epsilon_{1}\left(1-u_{2}\right)-\epsilon_{2} x_{2}^{2} \geq 0,
$$

where $\epsilon_{1}$ and $\epsilon_{2}$ are positive constants (we set $\epsilon_{1} \geq 0.1, \epsilon_{2} \geq 0.1$ ). Positive definiteness holds as

$$
\epsilon_{1}\left(1-u_{2}\right)+\epsilon_{2} x_{2}^{2}=\epsilon_{1}\left(1-\cos x_{1}\right)+\epsilon_{2} x_{2}^{2}
$$

is a positive definite function in the $\left(x_{1}, x_{2}\right)$-space (assuming all $x_{1}$ that differ by $2 \pi$ are in the same equivalence class).

An example of Lyapunov function for this whirling pendulum system, found using the sum of squares procedure, is given by

$$
V=0.33445 x_{2}^{2}+1.4615 u_{1}^{2}+1.7959 u_{2}^{2}-6.689 u_{2}+4.8931 .
$$




\subsection{Example 4.3: System with An Irrational Power Vector Field}

Enzymatic reactions that are described by Michaelis-Menten type equations [7] usually contain terms with non-integer powers. Here we give an example of how such systems can be analyzed. Consider a simple one dimensional system:

$$
\dot{x}=x^{\alpha}-1, \quad x \in \mathbb{R}_{+},
$$

where $\alpha$ is a parameter. The linearisation of this system about the equilibrium $x=1$ is $\dot{x}=\alpha x$ which implies that the system is locally stable for $\alpha<0$. Let us make a transformation $y=x-1$ to the above system to put its equilibrium at the origin:

$$
\dot{y}=(y+1)^{\alpha}-1 .
$$

Further to this transformation, we introduce the transformation $z=(y+$ $1)^{\alpha}-1$ and embed the system into a second order system with a polynomial vector field and an equality constraint that projects it back to 1-D:

$$
\begin{aligned}
& \dot{y}=z \\
& \dot{z}=\alpha \frac{(z+1) z}{y+1} \\
& z=(y+1)^{\alpha}-1 .
\end{aligned}
$$

The non-polynomial equality constraint (38) cannot be imposed in the sum of squares program in a similar manner as before. To proceed with the analysis, we will try to prove stability of the two dimensional system without the equality constraint, but keeping in mind that the system is, at the end of the day, one-dimensional. We will attempt to prove stability for

$$
\begin{aligned}
\alpha-\alpha_{h} & \leq 0 \\
-y+y_{l} & \leq 0 \\
-z+z_{l} & \leq 0 .
\end{aligned}
$$

We set $\alpha_{h}=-0.1$ and $y_{l}=-0.9$. This dictates that $z \geq\left(y_{l}+1\right)^{\alpha_{h}}-1 \triangleq z_{l}$. We search for a 4 th order Lyapunov function in $y, z$ but we do not require $V$ to be positive definite in both $y$ and $z$, by constructing $\phi(y, z)$ in $(21)$ appropriately. In particular the two Lyapunov conditions become:

$$
\begin{aligned}
& V(y, z ; \alpha)-\phi(y, z) \geq 0, \\
& -\frac{\partial V}{\partial y} \dot{y}-\frac{\partial V}{\partial z} \dot{z} \leq 0,
\end{aligned}
$$

for $\alpha, y, z$ satisfying (39)-(41), and additionally where

$$
\begin{aligned}
& \phi(y, z)=\epsilon_{1} y^{2}+\epsilon_{2} y^{4}+\epsilon_{3} z^{2}+\epsilon_{4} z^{4}, \\
& \sum_{i=1}^{4} \epsilon_{i} \geq 0.01, \quad \epsilon_{i} \geq 0, \forall i=1, \ldots, 4 .
\end{aligned}
$$




\begin{tabular}{|c|c|c|}
\hline Parameter & Units & Nominal Value \\
\hline$F / V$ & $\mathrm{hr}^{-1}$ & 1 \\
$k_{0}$ & $\mathrm{hr}^{-1}$ & $9703 \times 3600$ \\
$-\Delta H$ & $\mathrm{kcal} / \mathrm{kgmol}$ & 5960 \\
$\Delta E$ & $\mathrm{kcal} / \mathrm{kgmol}$ & 11843 \\
$\rho c_{p}$ & $\mathrm{kcal} /\left(\mathrm{m}^{3}{ }^{\circ} \mathrm{C}\right)$ & 500 \\
$T_{f}$ & ${ }^{\circ} \mathrm{C}$ & 25 \\
$C_{A_{f}}$ & $\mathrm{kgmol} / \mathrm{m}^{3}$ & 10 \\
$U A / V$ & $\mathrm{kcal} /\left(\mathrm{m}^{3}{ }^{\circ} \mathrm{C} \mathrm{hr}\right)$ & 150 \\
$T_{j}$ & ${ }^{\circ} \mathrm{C}$ & 25 \\
\hline
\end{tabular}

Table 1. Parameter values for the CSTR.

The inequality constraints (39)-(41) can be adjoined to the two conditions and a sum of squares program can be written using SOSTOOLS as in the previous examples. Indeed, such a Lyapunov function was constructed which allows for stability to be concluded.

\subsection{Example 4.4: Diabatic Continuous Stirred Tank Reactor}

Chemical reactors are the most important unit operation in a chemical process. In this section we consider the analysis of the dynamics of a perfectly mixed, diabatic, continuously stirred tank reactor (CSTR) [1]. We also assume a constant volume - constant parameter system for simplicity.

The reaction taking place in the CSTR is a first-order exothermic irreversible reaction $A \rightarrow B$. After balancing mass and energy, the reactor temperature $T$ and the concentration of species $A$ in the reactor $C_{A}$ evolve as follows:

$$
\begin{aligned}
\dot{C}_{A} & =\frac{F}{V}\left(C_{A_{f}}-C_{A}\right)-k_{0} e^{-\frac{\Delta E}{R T}} C_{A} \\
\dot{T} & =\frac{F}{V}\left(T_{f}-T\right)-\frac{\Delta H}{\rho c_{p}} k_{0} e^{-\frac{\Delta E}{R T}} C_{A}-\frac{U A}{V \rho c_{p}}\left(T-T_{j}\right)
\end{aligned}
$$

where $F$ is the volumetric flow rate, $V$ is the reactor volume, $C_{A_{f}}$ is the concentration of $A$ in the freestream, $k_{0}$ is the pre-exponential factor of Arrhenius law, $\Delta E$ is the reaction activation energy, $R$ is the ideal gas constant, $T_{f}$ is the feed temperature, $-\Delta H$ is the heat of reaction (exothermic), $\rho$ is the density, $c_{p}$ is the heat capacity, $U$ is the overall heat transfer coefficient, $A$ is the area for heat exchange, and $T_{j}$ is the jacket temperature. For the analysis, we use the values shown in Table 1.

The equilibrium of the above system is given by $\left(C_{A_{0}}, T_{0}\right)=(8.5636,311.171)$. We employ the following transformation: $x_{1}=C_{A} / C_{A_{0}}-1, x_{2}=T / T_{0}-1$; this serves two purposes: firstly it moves the equilibrium to the origin, and secondly it rescales the state to avoid numerical ill-conditioning. The transformed system then becomes: 


$$
\begin{aligned}
& \dot{x}_{1}=\frac{F}{V}\left(\frac{C_{A_{f}}}{C_{A_{0}}}-\left(x_{1}+1\right)\right)-k_{0} e^{-\frac{\Delta E}{R T_{0}\left(x_{2}+1\right)}}\left(x_{1}+1\right) \\
& \dot{x}_{2}=\frac{F}{V}\left(\frac{T_{f}}{T_{0}}-\left(x_{2}+1\right)\right)-\frac{\Delta H C_{A_{0}}}{\rho c_{p} T_{0}} k_{0} e^{-\frac{\Delta E}{R T_{0}\left(x_{2}+1\right)}}\left(x_{1}+1\right)-\frac{U A}{V \rho c_{p}}\left(\left(x_{2}+1\right)-\frac{T_{j}}{T_{0}}\right)
\end{aligned}
$$

Note that the system has an exponential term; the recasting will yield an indirect constraint, as discussed in Section 3. Define the state $x_{3}=e^{\frac{\Delta E x_{2}}{R T_{0}\left(x_{2}+1\right)}}-$ 1. Then an extra equation in the analysis would be

$$
\dot{x}_{3}=\frac{\Delta E}{R T_{0}\left(x_{2}+1\right)^{2}}\left(x_{3}+1\right) \dot{x}_{2}
$$

under the constraint that $x_{3}>-1$.

Then the full system, after we use the equilibrium relationship simplifies to:

$$
\begin{aligned}
& \dot{x}_{1}=-\frac{F}{V} x_{1}-k_{0} e^{-\frac{\Delta E}{R T_{0}}}\left(x_{1} x_{3}+x_{1}+x_{3}\right) \\
& \dot{x}_{2}=-\frac{F}{V} x_{2}-\frac{\Delta H C_{A_{0}}}{\rho c_{p} T_{0}} k_{0} e^{-\frac{\Delta E}{R T_{0}}}\left(x_{1} x_{3}+x_{1}+x_{3}\right)-\frac{U A}{V \rho c_{p}} x_{2} \\
& \dot{x}_{3}=\frac{\Delta E}{R T_{0}\left(x_{2}+1\right)^{2}}\left(x_{3}+1\right) \dot{x}_{2}
\end{aligned}
$$

This system is now of the form (10)-(11), with $\tilde{x}_{1}=\left(x_{1}, x_{2}\right)$ and $\tilde{x}_{2}=x_{3}$. To proceed, we define the set $\mathcal{D}_{1}$ as:

$$
\mathcal{D}_{1}=\left\{\left(x_{1}, x_{2}\right) \in \mathbb{R}^{2}:\left|x_{1}\right| \leq \gamma_{1},\left|x_{2}\right| \leq \gamma_{2}\right\}
$$

and then define the set $\mathcal{D}_{2}$ as

$$
\mathcal{D}_{2}=\left\{x_{3} \in \mathbb{R}:\left(x_{3}-e^{\frac{-\Delta E \gamma_{2}}{R T_{0}\left(-\gamma_{2}+1\right)}}-1\right)\left(x_{3}-e^{\frac{\Delta E \gamma_{2}}{R T_{0}\left(\gamma_{2}+1\right)}}-1\right) \leq 0\right\}
$$

Then the system is ready for analysis as per Proposition 4. For $\gamma_{1}=0.12$ and $\gamma_{2}=0.05$ a quartic Lyapunov function can be constructed for the system described by Equations (44)-(46) using Proposition 4. Here the following $\phi(x)$ is used:

$$
\phi(x)=\sum_{i=1}^{2} \sum_{j=2,4} \epsilon_{i, j} x_{i}^{j}+\sum_{j=1}^{4} \epsilon_{3, j} x_{3}^{j},
$$

with

$$
\begin{aligned}
\epsilon_{1,2}+\epsilon_{1,4}-0.1 & \geq 0 \\
\epsilon_{2,2}+\epsilon_{2,4}+\epsilon_{3,1}+\epsilon_{3,2}+\epsilon_{3,3}+\epsilon_{3,4}-0.1 & \geq 0 .
\end{aligned}
$$

The level curves of the constructed Lyapunov function are shown in Figure 3. 


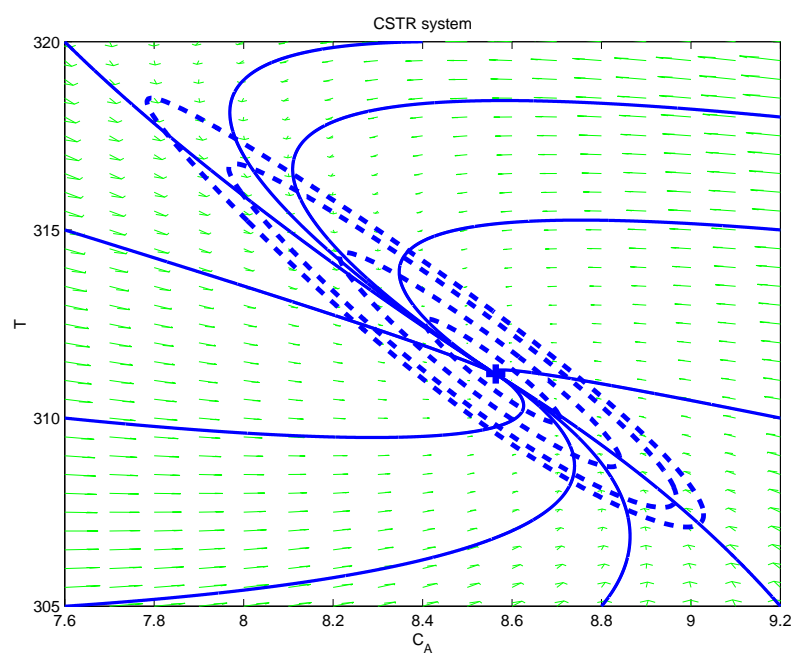

Fig. 3. Lyapunov function level curves for the system (42)-(43)

\section{Conclusions}

In this chapter we have presented a methodology to analyze systems described by non-polynomial vector fields using the sum of squares decomposition and a recasting procedure. Using this recasting procedure, a non-polynomial system can be converted into a rational form. An extension of the Lyapunov theorem in conjunction with the sum of squares decomposition and semidefinite programming can then be used to investigate the stability of the recasted system, the result of which can be used to infer the stability of the original system. Some examples of systems whose vector fields contain radical, trigonometric, irrational power, and exponential terms have been presented to illustrate the use of the proposed approach.

\section{Acknowledgements}

The authors would like to acknowledge the input of Professors Michael Savageau and John C. Doyle.

\section{References}

1. B. W. Bequette. Process Dynamics. Modeling, Analysis and Simulation. Prentice Hall, 1998.

2. J. Bochnak, M. Coste, and M.-F. Roy Real Algebraic Geometry. Springer-Verlag, Berlin, 1998. 
3. S. Boyd, L. El Ghaoui, E. Feron, and V. Balakrishnan. Linear Matrix Inequalities in System and Control Theory. Society for Industrial and Applied Mathematics (SIAM), 1994.

4. K. Furuta, M. Yamakita, and S. Kobayashi. Swing-up control of inverted pendulum using pseudo-state feedback. Journal of Systems and Control Engineering, 206:263-269, 1992

5. W. Hahn. Stability of Motion. Springer-Verlag, New York, 1967.

6. J. Marsden and T. Ratiu. Introduction to Mechanics and Symmetry. SpringerVerlag, NY, second edition, 1999.

7. J. D. Murray. Mathematical Biology. Springer-Verlag, second edition, 1993.

8. K. G. Murty and S. N. Kabadi. Some NP-complete problems in quadratic and nonlinear programming. Mathematical Programming, 39:117-129, 1987.

9. A. Papachristodoulou and S. Prajna. On the construction of Lyapunov functions using the sum of squares decomposition. In Proceedings of the 41 st IEEE Conf. on Decision and Control, 2002.

10. P. A. Parrilo. Structured Semidefinite Programs and Semialgebraic Geometry Methods in Robustness and Optimization. PhD thesis, Caltech, Pasadena, CA, 2000. Available at http://www.control.ethz.ch/ parrilo/pubs/index.html.

11. P. A. Parrilo and B. Sturmfels. Minimizing polynomial functions. In Workshop on Algorithmic and Quantitative Aspects of Real Algebraic Geometry in Mathematics and Computer Science, March, 1998.

12. S. Prajna, A. Papachristodoulou, and P. A. Parrilo. Introducing SOSTOOLS: A general purpose sum of squares programming solver. In Proceedings of the 41st IEEE Conf. on Decision and Control, 2002. Available at http://www.cds.caltech.edu/sostools and http://www . aut.ee.ethz.ch/ parrilo/sostools.

13. B. Reznick. Some concrete aspects of Hilbert's 17th problem. In Contemporary Mathematics, volume 253, pages 251-272. American Mathematical Society, 2000.

14. M. A. Savageau and E. O. Voit. Recasting nonlinear differential equations as S-systems: a canonical nonlinear form. Mathematical Biosciences, 87(1):83-115, 1987.

15. J. F. Sturm. Using SeDuMi 1.02, a MATLAB toolbox for optimization over symmetric cones. Optimization Methods and Software, 11-12:625-653, 1999. Available at http://fewcal.kub.nl/sturm/software/sedumi.html.

16. L. Vandenberghe and S. Boyd. Semidefinite programming. SIAM Review, 38(1):49-95, 1996.

17. V. A. Yakubovic. S-procedure in nonlinear control theory. Vestnik Leningrad University, 4(1):73-93, 1977. English translation.

18. V. Zubov. Methods of A.M. Lyapunov and Their Application. P. Noordhoff Ltd, Groningen, The Netherlands, 1964. 